\title{
Influence of boron doping on the dynamics of formation of Os metal nanoclusters on graphitic surfaces
}

\author{
Anaïs Pitto-Barry and Nicolas P. E. Barry* \\ Received (in $X X X, X X X)$ Xth $X X X X X X X X X 20 X X$, Accepted $X t h X X X X X X X X X 20 X X$ \\ ${ }_{5}$ DOI: $10.1039 / \mathbf{b 0 0 0 0 0 0 x}$
}

The fabrication of osmium nanoclusters from single atoms has been studied in real-time on B-doped and B-free graphitic surfaces. The dynamics of nucleation on both surfaces are identified, captured, and reported. The nucleation is $\mathrm{ca}$. 2× 10 faster on B-doped surface compared to the B-free surface (38 $\mathrm{pm} \cdot \mathrm{min}^{-1}$ versus $18 \mathrm{pm} \cdot \mathrm{min}^{-1}$ ), suggesting osmium-boron interactions within the nanomaterials.

Metal nanocrystals have a number of potential applications, ${ }^{1-6}$ particularly in electronics, ${ }^{7}$ healthcare, ${ }^{8-10}$ and catalysis. ${ }^{11,} 12$ The

15 dimensions of such nanocrystals have a direct influence on their physical and chemical properties. ${ }^{13}$ Control over their nucleation rates is therefore of paramount importance for the synthesis of nanocrystals of well-defined sizes and geometries. However, the process of nucleation is not only experimentally challenging to 20 image at the atomic level, but also greatly depends on the matrix supporting the nanocrystal formation.

We recently showed that electron irradiation of metal complexes encapsulated into micelles made of polymers leads to the generation of graphitic surfaces on which single atoms assemble 25 to produce nanoclusters. ${ }^{14-17}$ This methodology was used to synthesise precious metal nanoclusters ( $\mathrm{Ru}, \mathrm{Os}, \mathrm{Ir}, \mathrm{Au})$, as small as $15 \AA$ in diameter in less than 1 hour, as well as to allow the imaging, in real-time, of their nucleation steps. ${ }^{18}$ The versatility of this methodology also provides space for chemical 30 modifications of the micelle precursors. We took advantage of this versatility to produce $\mathrm{B} / \mathrm{S}$-doped and $\mathrm{B} / \mathrm{Se}$-doped matrixes and reported that Os atoms moved $c a .26 \times$ faster on the $\mathrm{B} / \mathrm{Se}$ surface compared to the $\mathrm{B} / \mathrm{S}$ surface. ${ }^{15}$

This methodology was made possible by the dramatic progress 35 made in the design and structure of electron microscopes. The new generations of microscopes possess atomic resolution capabilities and ultra-fast cameras. Dynamic processes, such as nucleation and nanocrystal growth, can therefore be imaged in real time, ${ }^{19-23}$ while the high-energy electron beam can be utilised 40 to structurally modify nanomaterials. ${ }^{24-32}$

Using this methodology, we report here the fabrication of osmium nanoclusters on a novel boron-free and sulfur-doped matrix, obtained by changing the osmium-coordinated ligand in the micelle precursors. Furthermore, the aggregation of single 45 atoms on such S-doped graphitic surface to form nanoclusters are captured and their nucleation rate are studied. These experimental data are compared with the ones obtained on a B/S-doped matrix, providing new insights into boron-osmium interactions at the atomic level.
50

We prepared two graphitic surfaces. The first was doped with hetero-atoms boron and sulfur $(\mathrm{B} / \mathrm{S})$ according to the procedure we described previously, using $\left[\operatorname{Os}\left(\eta^{6}-p\right.\right.$-cymene $)(1,2$-dicarbacloso-dodecarborane-1,2-dithiolato)] encapsulated in the ${ }_{55}$ Pluronic ${ }^{\circledR}$ P123 block-copolymer. ${ }^{16}$ The resulting OsMs micelles were then deposited as aqueous droplets $([\mathbf{O s M s}]=1 \mathrm{mg} / \mathrm{mL})$ onto lacey carbon-coated grids to produce an unsupported film over the grid holes for examination by aberration-corrected high resolution transmission electron microscope (AC-TEM) with a ${ }_{60}$ Schottky thermal field-emission source $\left(80 \mathrm{keV} ; 1.9 \mathrm{pA} \mathrm{cm}^{-2}\right.$ or $7.6 \times 10^{7}$ electrons $\mathrm{nm}^{-2} \mathrm{~s}^{-1}$ ). The second graphitic surface, only doped with sulfur, was prepared by irradiating micelles made of [Os $\left(\eta^{6}-p\right.$-cymene)(benzene-1,2-dithiolato)] encapsulated in the tri-block copolymer P123 (OsMs2; see Materials and methods), ${ }_{65}$ following a similar strategy. This simple structural modification of the encapsulated metal complex allows for the introduction, or not, of boron atoms into the graphitic matrix produced by electron irradiation of the metallated micelles (Figure 1).

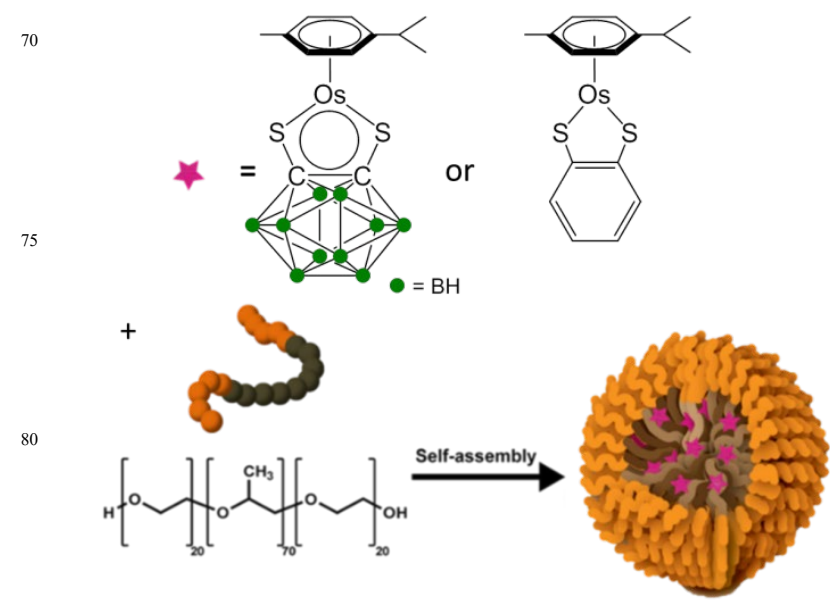

85 Figure 1. Self-assembly of block copolymer micelles containing the encapsulated Os metal complexes.

The TEM grid supporting the OsMs2 micelles was irradiated for up to 70 minutes. As with OsMs, structural changes were observed within the Pluronic ${ }^{\circledR}$ film formed by the spreading of ${ }_{90}$ OsMs2 within the holes of lacey carbon TEM grids upon irradiation with the high-energy electron beam. A turbostratic graphitic structure was formed in less than 5 minutes. Concomitantly, the osmium complexes were decomposed, and 
precious metal nanoclusters were imaged after 10 min (Figures 2 and 3).

Electron energy loss spectroscopy (EELS) confirmed the 5 presence of the Os atoms, but also suggested that sulfur from the benzene ligand are present throughout the graphitic matrix suggesting the formation of an S-doped graphitic matrix (Figure 2). Importantly, when the $\mathrm{B} / \mathrm{S}$-doped graphitic surface was initially studied, ${ }^{16}$ sites with Os clusters were identified to be 10 close to both high-boron and high-sulfur sites, therefore suggesting that the B-atoms were still present in the surface and had not been removed by a knock-on effect of the e-beam.
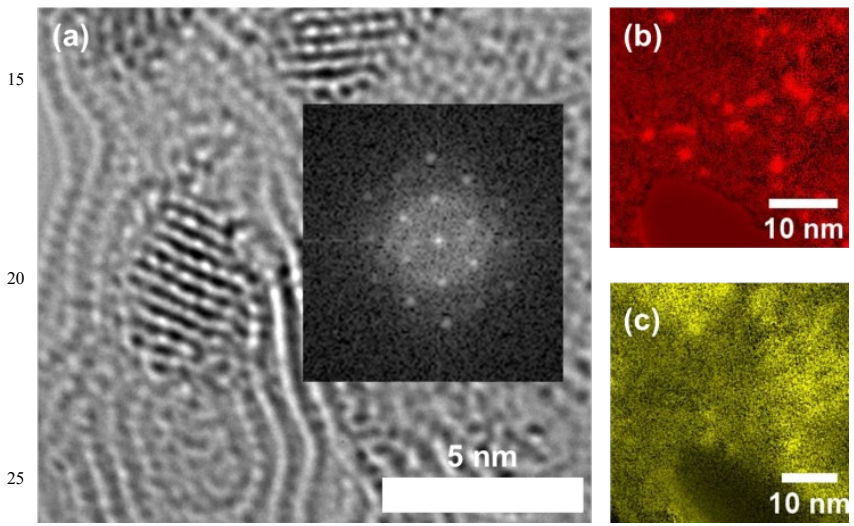

Figure 2. (a) AC-TEM image and FFT of an Os nanocrystal on a Bfree/S-doped turbostratic graphitic structure obtained after $50 \mathrm{~min}$ electron beam irradiation. Scale bar $5 \mathrm{~nm}$. (b) Electron energy loss

30 elemental mapping of nanocrystals and self-supported matrix, osmium $\mathrm{O}$ map, (c) sulfur L map.

The crystallinity of the biggest osmium nanoclusters generated in situ by electron irradiation of the B-free/S-doped surface was confirmed by Fast Fourier Transform (FFT) analysis (Figure 2 for 35 the FFT of a ca. $4.5 \mathrm{~nm}$-diameter Os nanocrystal). This observation is in accordance with the results obtained with $\mathrm{Ru}$, Os, Ir, Au crystals on the B/S-doped surface. ${ }^{18}$ Interestingly it not only generalises the methodology to the new B-free/S-doped surface, but also demonstrates that the carborane ligand is not 40 needed to generate the graphitic surface. This is of significant importance for the generalisation of our methodology. Indeed, although carborane derivatives introduce unusual reactivities and peculiar chemistry, the benzene-1,2-dithiolato ligand is a more readily available, easier to functionalise, and cheaper ligand than 45 carboranes. This opens-up a new avenue for the fabrication of precious metal nanoclusters using this methodology. Indeed, we have a significant interest in the synthesis of electron-deficient metal complexes designed around this benzene-1,2-dithiolato scaffold, ${ }^{33}, 34$ and this will open new perspectives for the ${ }_{50}$ introduction of a range of new doping elements, such as $\mathrm{Cl}, \mathrm{Br}, \mathrm{P}$, and N-doping elements.

We then imaged the early steps of nanocluster aggregation on both B-free/S-doped and B/S-doped graphitic surfaces (Table 1,

${ }_{55}$ Figure 3 ). The metal-metal distances in the osmium nanoclusters were measured on aggregates as small as a few atoms on their surface. The average Os-Os distances determined for 30 different clusters per irradiation time were found to be close to those in the bulk (Table 1). More importantly, the presence or absence of ${ }_{60}$ boron atoms does not seem to have a significant influence on the metal-metal distances. This is however only indicative owing to the three-dimensional structure of the crystals, as well as their ability to roll on the graphitic matrixes. As found previously on the B/S-doped surface, no change in the metal-metal distances 65 were observed during the nanocluster growth.

Table 1 Osmium nanocluster growth parameters.

\begin{tabular}{lccc}
\hline Surface & \multicolumn{2}{c}{ M-M distance $(\mathrm{nm})$} & $\begin{array}{c}\text { Growth rate } \\
\left(\mathrm{pm} \cdot \mathrm{min}^{-1}\right)\end{array}$ \\
\hline & Nanocluster & Bulk $^{30,31}$ & \\
\hline B-free/S-doped & $0.278 \pm 0.03$ & 0.2705 & 38.0 \\
B/S-doped & $0.263 \pm 0.04$ & 0.2705 & 18.5 \\
\hline
\end{tabular}

\begin{tabular}{|l|l|}
\hline $10 \mathrm{~min}$ \\
\hline
\end{tabular}
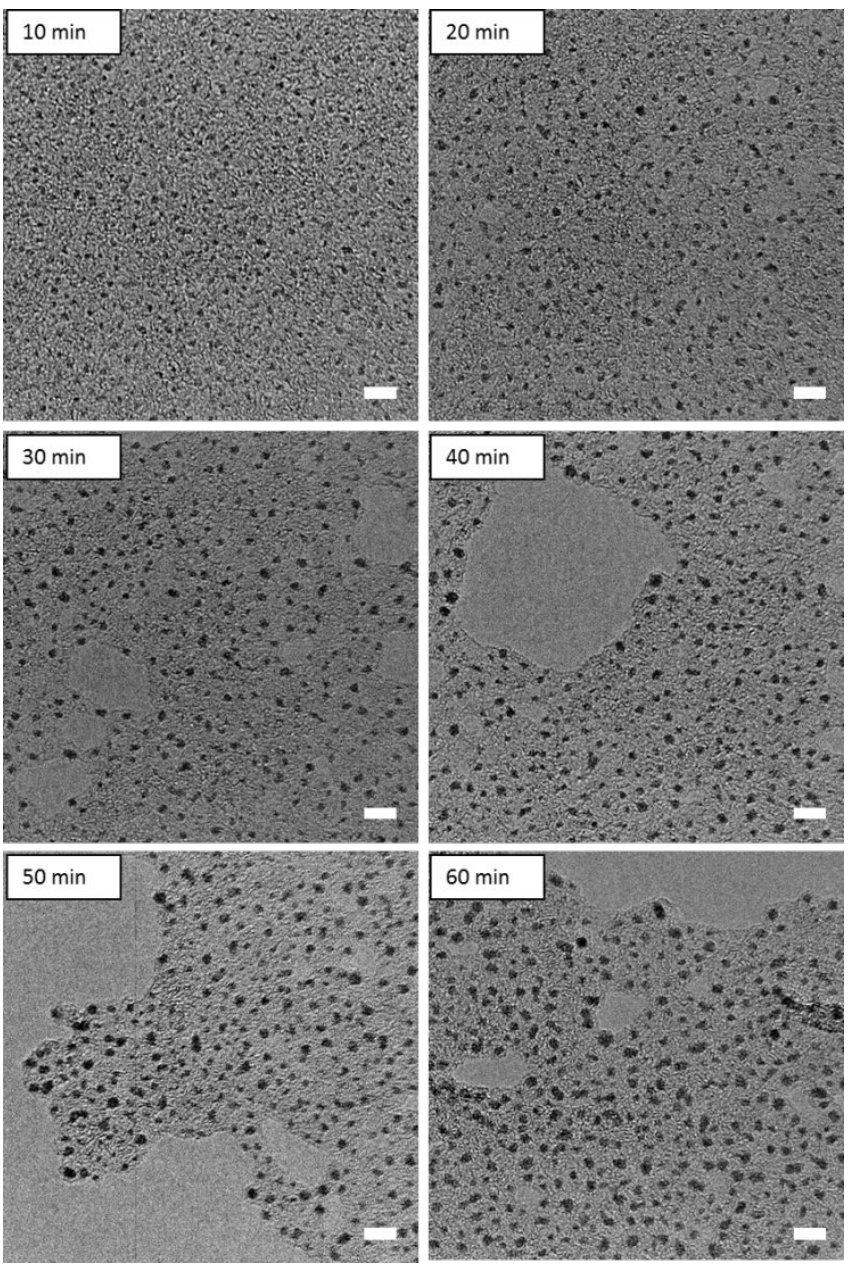

Fig. 3 Linear growth of Os nanoclusters on the B-free/S-doped surface. Scale bar $10 \mathrm{~nm}$.

We then measured the length of the osmium clusters over time and found on both graphitic matrixes a linear relationship between growth and time, with narrow size distributions as can be seen by the error bars in Figure 4 (representing the standard 75 deviations from measurements on at least 30 clusters at each time point).

The growth mechanism is consistent with our previous observations, ${ }^{14-17}$ and is believed to be via migration and 
coalescence of individual atoms and small clusters rather than by the kinetics of diffusion and Ostwald ripening (since no evidence of atoms leaving the nanoclusters was observed). The linear relationship between growth and time does suggest that the size 5 of the clusters can be controlled by modulation of the time of irradiation. However, surprisingly, the nucleation apparent growth rate was found to be $c a .2$ times faster on the B-free/Sdoped surface than on the B/S-doped surface $\left(38 \mathrm{pm} \cdot \mathrm{min}^{-1}\right.$ versus $\left.18.5 \mathrm{pm} \cdot \mathrm{min}^{-1}\right)$. These results suggest that the boron doping 10 appears to create anchoring sites for osmium atoms and contribute to the control of their migration such that it is within the timescale of imaging, although the effect of Se- versus Sdoping seems to have a greater influence on the nucleation rate than the presence or absence of boron atoms (26 times faster on 15 Se-doped surfaces than on S-doped surfaces).

It is however clear that both rates are within the same order of magnitude and this is also consistent with our previous observations on the sulfur versus selenium doping effects. We hypothesise that the relatively narrow differences of crystal 20 growth observed on the two surfaces may be explained by the fact that the kinetic energy of the electron beam is the main driving force for the observed dynamic of osmium atoms in the TEM chamber. Nonetheless, these results also suggest the presence of unprecedented metal-boron interactions within the 25 nanomaterials. These results also indicate that hetero-atoms can act as trapping sites for individual metal atoms, slowing their motions on the surface, with a rate-dependency related to the nature of the metal and the doping atoms.
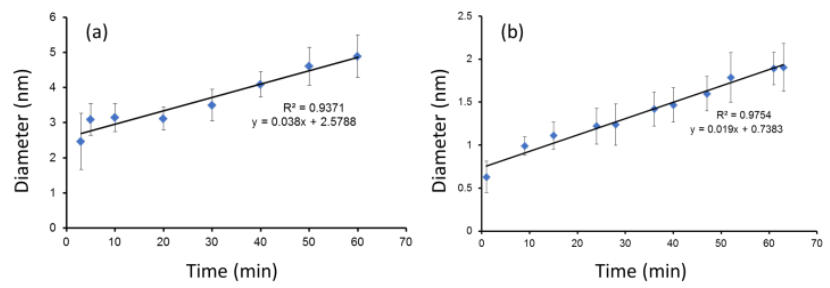

30 Fig. 4 Width of the nanoclusters versus irradiation time on (a) B-free/Sdoped and (b) B/S-doped graphitic surfaces. The error bars are the standard deviations from measurements on at least 30 clusters at each time point.

\section{Conclusions}

${ }_{35}$ Recent advances in the design and capabilities of electron microscopy allow the dual use of TEM as an analytical tool for the study of nucleation, and as an external force to structurally modify nano-objects. Here, we have used these capabilities to generate single osmium atoms and to fabricate osmium 40 nanoclusters on B-free/S-doped and B/S-doped graphitic surfaces by electron irradiation of triblock copolymer micelles containing encapsulated metal complexes $\left[\mathrm{Os}\left(\eta^{6}-p\right.\right.$-cymene)(1,2-dicarbacloso-dodecarborane-1,2-dithiolato $)] \quad$ or $\quad\left[\mathrm{Os}\left(\eta^{6}-p\right.\right.$ cymene)(benzene-1,2-dithiolato)]. The boron-doping appears to 45 create anchoring sites for individual osmium atoms, slowing the nucleation apparent growth rate (by a factor 2). Direct interactions between osmium and boron appear to be unknown in nanomaterials. The development of a new family of organometallic precursors based on the simple benzene moiety 50 will allow the facile introduction of a range of doping heteroatoms on such graphitic surfaces and may offer potential for the control of dynamics of metal atoms on fabricated matrices.

\section{Materials and methods}

Materials. The preparations of the complexes [Os $(p$-cym)(1,2${ }_{55}$ dicarba-closo-dodecaborane-1,2-dithiolato $\left.)\right]$, and $\left[\mathrm{Os}\left(\eta^{6}-p\right.\right.$ cymene)(benzene-1,2-dithiolato)], were based on previous reports. ${ }^{16,} 35,36$ The triblock copolymer P123 [poly(ethylene glycol)-block-poly(propylene glycol)-block-poly(ethylene glycol)] was purchased from Sigma-Aldrich. The micellar 60 formulation OsMs was prepared according to previous methodology. ${ }^{35}$ Anhydrous tetrahydrofuran (Aldrich) was used. 18.2 $\mathrm{M} \Omega \cdot \mathrm{cm}$ purity water was collected from a Purelab ${ }^{\circledR}$ UHQ USF Elga system. Holey carbon grids with 200 mesh and lacey carbon grids were purchased from Quantifoil Micro Tools Gmbh 65 and Elektron Technology UK Ltd, respectively. Synthesis of $\boldsymbol{O} \boldsymbol{S M} \boldsymbol{s}_{2}$. A tetrahydrofuran (THF) solution $(1 \mathrm{~mL})$ of $\left[\mathrm{Os}\left(\eta^{6}-p\right.\right.$ cymene)(benzene-1,2-dithiolato)] complex $(5 \mathrm{mg} / \mathrm{mL})$ was added to an aqueous solution $(10 \mathrm{~mL})$ of polymer P123 $(5 \mathrm{mg} / \mathrm{mL})$ and the resultant mixture was stirred at ambient temperature for $4 \mathrm{~h}$.

70 The solution was then dialysed to remove the THF $(\mathrm{MWCO}=$ $1000 \mathrm{Da}$ ), for $48 \mathrm{~h}$, and then freeze-dried. High resolution electron microscopy HR-TEM: A JEOL JEM-ARM200F HRTEM was operated at $80 \mathrm{keV}, 1.9 \mathrm{pA} \cdot \mathrm{cm}^{-2}$, with spherical aberration $\left(C_{\mathrm{s}}\right)$ tuned to approximately $+1 \mu \mathrm{m}$ and images were 75 recorded on a Gatan SC1000 Orius CCD camera. All the images were analysed with ImageJ (Fiji) software. To determine the diameter of the nanoclusters, the Fiji software was used (Fiji allowing the viewing of stacks of images, contrary to ImageJ). Only one co-author was responsible for determining all lengths 80 for all nanoclusters, by manually selecting the "start" and "end" of each nanocluster, and the graphs were plotted after all nanoclusters were measured in order to eliminate possible biases. The diameter for almost non-spherical nanoclusters is determined by assuming nanoclusters have a spherical shape. Nanoclusters 85 which exhibit a high anisotropy are not taken into account, however they are rare. Nanoclusters on the edge of the matrix are not taken into account either, because of possible folding of the matrix.

\section{Acknowledgments}

90 This project was supported by the Royal Society (University Research Fellowship no. UF150295 to NPEB) and by the Academy of Medical Sciences/the Wellcome Trust/the Government Department of Business, Energy and Industrial Strategy/the British Heart Foundation Springboard Award 95 [SBF003\1170 to NPEB].

\section{Notes and references}

School of Chemistry and Biosciences, University of Bradford, Bradford, BD7 1DP, United Kingdom; N.Barry@bradford.ac.uk

$1001 . \quad$ D.-W. Lim, J. W. Yoon, K. Y. Ryu and M. P. Suh, Angew. Chem. Int. Ed., 2012, 51, 9814-9817.

2. J.-S. Lee, M. V. Kovalenko, J. Huang, D. S. Chung and D. V. Talapin, Nat Nano, 2011, 6, 348-352.

3. Y. T. Zhu and X. Liao, Nat. Mater., 2004, 3, 351-352. 1054 P. Wu and X.-P. Yan, Chem. Soc. Rev., 2013, 42, 5489-5521. 


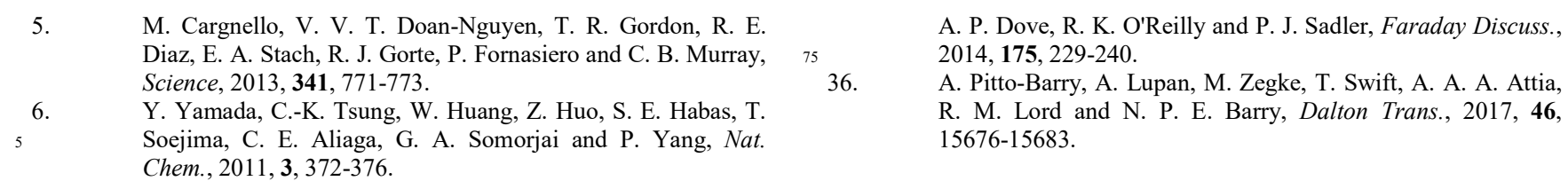

$7 . \quad$ D. Jariwala, V. K. Sangwan, L. J. Lauhon, T. J. Marks and M. C. Hersam, Chem. Soc. Rev., 2013, 42, 2824-2860.

8. E.-K. Lim, T. Kim, S. Paik, S. Haam, Y.-M. Huh and K. Lee, 10 Chem. Rev., 2014, DOI: 10.1021/cr300213b.

9. J. Yao, M. Yang and Y. Duan, Chem. Rev., 2014, 114, 61306178.

10. J. A. Hubbell and A. Chilkoti, Science, 2012, 337, 303-305.

11. B. Cornelio, G. A. Rance, M. Laronze-Cochard, A. Fontana, J.

15 Sapi and A. N. Khlobystov, J. Mater. Chem. A, 2013, 1, 87378744.

12. S. T. Skowron, T. W. Chamberlain, J. Biskupek, U. Kaiser, E. Besley and A. N. Khlobystov, Acc. Chem. Res., 2017, 50, $1797-1807$.

20 13. J. Jortner and C. N. R. Rao, Pure Appl. Chem., 2002, 74, 1491-1506.

14. A. Pitto-Barry, L. M. A. Perdigao, M. Walker, J. Lawrence, G. Costantini, P. J. Sadler and N. P. E. Barry, Dalton Trans., 2015, 44, 20308-20311.

$2515 . \quad$ N. P. E. Barry, A. Pitto-Barry, J. Tran, S. E. F. Spencer, A. M. Johansen, A. M. Sanchez, A. P. Dove, R. K. O'Reilly, R. J. Deeth, R. Beanland and P. J. Sadler, Chem. Mater., 2015, 27, 5100-5105.

16. N. P. E. Barry, A. Pitto-Barry, A. M. Sanchez, A. P. Dove, R. J. Procter, J. J. Soldevila-Barreda, N. Kirby, I. Hands-Portman, C. J. Smith, R. K. O'Reilly, R. Beanland and P. J. Sadler, Nat. Commun., 2014, 5, 3851.

17. A. Pitto-Barry, T. Geraki, M. D. Horbury, V. G. Stavros, J. F. W. Mosselmans, R. I. Walton, P. J. Sadler and N. P. E. Barry, 35 Chem. Commun., 2017, 53, 12898-12901.

18. A. Pitto-Barry, P. J. Sadler and N. P. E. Barry, Chem. Commun., 2016, 52, 3895-3898.

19. M. A. Lebedeva, T. W. Chamberlain, M. Schröder and A. N. Khlobystov, Chem. Mater., 2014, 26, 6461-6466.

$4020 . \quad$ P. Rodríguez-Zamora, F. Yin and R. E. Palmer, J. Phys. Chem. A, 2014, 118, 8182-8187.

21. Z. He, K. He, A. W. Robertson, A. I. Kirkland, D. Kim, J. Ihm, E. Yoon, G.-D. Lee and J. H. Warner, Nano Lett., 2014, 14, 3766-3772.

45 22. M. Ibáñez and A. Cabot, Science, 2013, 340, 935-936.

23. R. F. Egerton, Microsc. Microanal., 2013, 19, 479-486.

24. X. Qi, Y. Huang, M. Klapper, F. Boey, W. Huang, S. D. Feyter, K. Müllen and H. Zhang, J. Phys. Chem. C 2010, 114, 13465-13470.

$5025 . \quad$ J.-U. Kim, S.-H. Cha, K. Shin, J. Y. Jho and J.-C. Lee, J. Am. Chem. Soc., 2005, 127, 9962-9963.

26. A. H. Latham and M. E. Williams, Langmuir, 2008, 24, 14195-14202.

27. A. H. Latham, M. J. Wilson, P. Schiffer and M. E. Williams, $J$. 55 Am. Chem. Soc., 2006, 128, 12632-12633.

28. C. W. Huang, C. L. Hsin, C. W. Wang, F. H. Chu, C. Y. Kao, J. Y. Chen, Y. T. Huang, K. C. Lu, W. W. Wu and L. Chen, J. Nanoscale, 2012, 4, 4702-4706.

29. J. W. Liu, J. E. Xu, Y. Ni, F. J. Fan, C. L. Zhang and S. H. Yu, 60 ACS Nano 2012, 6, 4500-4507.

30. Z. W. Wang and R. E. Palmer, Phys. Rev. Lett., 2012, 108, 245502 .

31. J. A. Watts, M. W. Fay, G. A. Rance, P. D. Brown and A. N. Khlobystov, Carbon, 2018, 139, 538-544.

$6532 . \quad$ R. W. Lodge, G. A. Rance, M. W. Fay and A. N. Khlobystov, Nanoscale, 2018, 10, 19046-19051.

33. A. Pitto-Barry, A. Lupan, C. Ellingford, A. A. A. Attia and N. P. E. Barry, ACS Appl. Mater. Interfaces, 2018, 10, 1369313701 .

70 34. J. Zhang, A. Pitto-Barry, L. Shang and N. P. E. Barry, R. Soc. Open Sci., 2017, 4, 170786.

35. N. P. E. Barry, A. Pitto-Barry, I. Romero-Canelon, J. Tran, J. J. Soldevila-Barreda, I. Hands-Portman, C. J. Smith, N. Kirby, 